\title{
Strength Training Reduces Fat Accumulation and Improves Blood Lipid Profile Even in the Absence of Skeletal Muscle Hypertrophy in High-Fat Diet-Induced Obese Condition
}

\author{
Catarina Denise Entringer Contreiro $\left(\mathrm{D},{ }^{1}\right.$ Leonardo Carvalho Caldas $\left(\mathrm{D},{ }^{2}\right.$ \\ Breno Valentim Nogueira $\mathbb{D}^{3},^{3}$ André Soares Leopoldo $\mathbb{D}^{1,2}$ Ana Paula Lima-Leopoldo ${ }^{10},{ }^{1,2}$ \\ and Lucas Guimarães-Ferreira ${ }^{1}{ }^{1}$ \\ ${ }^{1}$ Graduate Program in Nutrition and Health, Center of Health Sciences, Federal University of Espirito Santo, \\ Vitória, ES, Brazil \\ ${ }^{2}$ Center of Physical Education and Sports, Federal University of Espírito Santo, Vitória, ES, Brazil \\ ${ }^{3}$ Center of Health Sciences, Federal University of Espírito Santo, Vitória, ES, Brazil
}

Correspondence should be addressed to André Soares Leopoldo; andre.leopoldo@ufes.br

Received 1 June 2020; Revised 14 October 2020; Accepted 30 October 2020; Published 21 November 2020

Academic Editor: Aron Weller

Copyright (c) 2020 Catarina Denise Entringer Contreiro et al. This is an open access article distributed under the Creative Commons Attribution License, which permits unrestricted use, distribution, and reproduction in any medium, provided the original work is properly cited.

\begin{abstract}
The aim was to investigate the effect of strength training on skeletal muscle morphology and metabolic adaptations in obese rats fed with unsaturated high-fat diet (HFD). The hypothesis was that strength training induces positive metabolic adaptations in obese rats despite impaired muscle hypertrophy. Male Wistar rats $(n=58)$ were randomized into two groups and fed a standard diet or a high-fat diet (HFD) containing $49.2 \%$ of fat. After induction and maintenance to obesity, the rats were divided into four groups: animals distributed in sedentary control (CS), control submitted to strength training protocol (CT), obese sedentary $(\mathrm{ObS})$, and obese submitted to strength training protocol (ObT). The exercise protocol consisted of 10 weeks of training on a vertical ladder (three times a week) with a load attached to the animal's tail. At the end of 10 weeks, strength training promoted positive changes in the body composition and metabolic parameters in obese animals. Specifically, ObT animals presented a reduction of $22.6 \%$ and $14.3 \%$ in body fat and adiposity index when compared to ObS, respectively. Furthermore, these rats had lower levels of triglycerides $(\mathrm{ObT}=23.1 \pm 9.5$ vs. $\mathrm{ObS}=30.4 \pm 6.9 \mathrm{mg} / \mathrm{dL})$ and leptin $(\mathrm{ObT}=13.2 \pm 7.2 v s . \mathrm{ObS}=20.5 \pm 4.3 \mathrm{ng} / \mathrm{mL})$. Training (ObT and CT) induced a greater strength gain when compared with the respective control groups. In addition, the weight of the flexor hallucis longus (FHL) muscle was higher in the ObT group than in the CT group, representing an increase of $26.1 \%$. However, training did not promote hypertrophy as observed by a similar cross-sectional area of the FHL and plantar muscles. Based on these results, high-intensity strength training promoted an improvement of body composition and metabolic profile in obese rats that were fed a high-fat diet without skeletal muscle adaptations, becoming a relevant complementary strategy for the treatment of obesity.
\end{abstract}

\section{Introduction}

The prevalence of overweight and obesity has been increasing rapidly and globally, being considered a public health problem by the World Health Organization [1]. Obesity is a chronic multifactorial disease characterized by increased adipose tissue, leading to health hazards [1]. It is considered a relevant risk factor for the development of chronic noncommunicable diseases, such as cardiovascular diseases and type 2 diabetes mellitus, in addition to being associated with increased morbidity and mortality rates and reduction in life expectancy [2-5].

Physical exercise is indicated as an efficient strategy in the prevention and treatment of obesity, especially in obesity caused by high caloric intake and sedentary lifestyle $[6,7]$. The benefits of regular exercise are related to decreased 
adipose mass, increased muscle mass, and protection against diseases that are associated with obesity $[8,9]$. For gains of muscle strength and hypertrophy, strength training has been show to promote these adaptations in rodents and humans [10-14].

The American College of Sports Medicine recommends strength training as an auxiliary strategy in weight loss, aiming to improve the functional capacity by increasing the strength and muscle power and increasing the daily energy expenditure [8]. In addition, studies indicate that higher levels of muscular strength are associated with lower rates of risk factors associated with obesity and mortality [15-17]. Recent research has shown impairment in regeneration capacity of skeletal muscle in response to strength exercises in obese rats [18]. It is well known that muscle overload, as seen during strength training, leads to the activation of cell signaling pathways involved with muscle protein synthesis $[19,20]$. In previous studies, inhibition of the Akt/mTOR pathway abolished muscle hypertrophy in a surgical overload model in mice [21]. Obesity induced by high-fat diet can lead to disruption of the Akt pathway and insulin resistance in peripheral tissues such as skeletal muscles [22]. However, the effects of obesity on protein synthesis in skeletal muscle and muscle hypertrophy in response to overload are still not well understood [23].

In this context, the objective of this current study was to evaluate the effect of strength training on skeletal muscle morphology and on metabolic adaptations in obese rats. The hypothesis was that strength training induces positive metabolic adaptations in obese rats despite impaired muscle hypertrophy.

\section{Materials and Methods}

2.1. Animals and Treatment. Fifty-eight male Wistar rats, with 30 days of age and initial weight of approximately $150 \mathrm{~g}$, were used. The animals were kept in individual cages in controlled environment with temperature at $24 \pm 2^{\circ} \mathrm{C}$, humidity $55 \pm 5 \%$, and reversed light/dark cycle of 12 hours. All procedures used in the study were approved by the Ethics Committee on the Use of Animals (CEUA) of the Federal University of Espírito Santo (protocol number 16/2016).

2.2. Food Consumption. The animals received $40 \mathrm{~g}$ of feed daily, and after 24 hours, the remaining noningested amount was measured. Control animals received standard diet, and obese animals received an unsaturated high-fat diet. Water was supplied ad libitum.

2.3. Composition of Diets. The standard diet presented a caloric content of $2.92 \mathrm{kcal} / \mathrm{g}, 13.9 \%$ of calories from fat, $55.9 \%$ from carbohydrates, and $30.2 \%$ from proteins (Nuvilab CR1-Nuvital ${ }^{\circledR}$, Colombo, Paraná, Brazil). In turn, the high-fat diet presented a caloric content of $3.65 \mathrm{kcal} / \mathrm{g}$, $49.2 \%$ of calories from fat, $28.9 \%$ from carbohydrates, and 21.9\% from proteins (RC Focus 2413, 2414, 2415, and 2416, Agroceres $^{\circledR}$, Rio Claro, São Paulo, Brazil). Animals fed with high-fat diet received a four-flavor cycle of the diet, in which the nutritional composition was the same except for the flavoring additives (cheese, bacon, chocolate, and vanilla). The high-fat diet was provided throughout the experimental protocol.

2.4. Experimental Design. After seven days of acclimatization, the animals were randomly distributed into two groups: (1) control (C, $n=29)$ and (2) obese (Ob, $n=29)$. The experimental protocol consisted of 26 weeks, divided into three moments, as shown in Figure 1. Moment 1 (M1) was the first two weeks of induction to obesity. When the mean body weight of experimental groups $\mathrm{C}$ and $\mathrm{Ob}$ presented statistical difference, the initial moment of obesity was characterized. After induction to obesity, the animals were kept under the same conditions for 14 consecutive weeks for maintenance of this condition, which we classified as moment 2 (M2). The training protocol, carried out for 10 weeks, determined moment 3 (M3).

2.5. Redistribution of Groups. After 14 weeks of period for induction to obesity, the animals were further distributed into two groups according to a separation point (SP) between the $\mathrm{C}$ and $\mathrm{Ob}$ groups. This criterion was adopted in order to constitute homogeneous groups; a 95\% confidence interval was constructed based on the mean values of body fat (BF) obtained after euthanasia [24]. The SP consists of a midpoint between the upper limit from the $\mathrm{C}$ group and the lower limit of the $\mathrm{Ob}$ group. From this point, the control animals with a body fat above the SP and the $\mathrm{Ob}$ animals with a BF below the SP were excluded from the $\mathrm{C}$ and $\mathrm{Ob}$ groups, respectively, ensuring the homogeneity of the treated and control groups. This criterion was adopted because in biological experimentation misclassification can occur; in other words, animals submitted to high-fat diets should be classified as obese even though they may exhibit characteristics of control animals. Therefore, animals submitted to different diet models do not always present the expected response. This fact can lead to erroneous animal classification and, consequently, false conclusions in several studies involving obesity models. Based on this criterion, the groups were redistributed for the purpose of having obese prone animals and controls. Thus, 29 rats from each group were classified as false controls $(n=4)$ and resistant to obesity $(n=8)$ and, therefore, were excluded from this study. It is important to highlight that six animals died $(\mathrm{CS}=2$, $\mathrm{CT}=1$, and $\mathrm{ObS}=3$ ) from undetermined causes throughout the experimental protocol and four rats did not perform the proposed physical training in $\mathrm{ObT}(n=2)$ and CTF $(n=2)$.

In $\mathrm{M} 2$, animals were redistributed into two more groups as absence or presence of strength training (RT). Therefore, after the training protocol, this study was composed of four groups: sedentary control (CS; $n=10)$, control submitted to strength training protocol (CT; $n=10)$, obese sedentary (ObS; $n=7$ ), and obese submitted to strength training protocol (ObT; $n=9)$. 


Moments
$\begin{aligned} & \text { Groups and } \\ & \text { distribution }\end{aligned}$

Figure 1: Schematic representation of the experimental design. Moment 1 (M1): 2 weeks for the induction period to obesity. After induction, the animals were kept under the same conditions for 14 consecutive weeks for maintenance of obesity, which was classified as moment 2 (M2). The training protocol, carried out for 10 weeks, determined moment 3 (M3).

2.6. Training Protocol. The CST and ObT groups were submitted to the strength training protocol performed in stair climbing adapted from the study by Hornberger and Farrar [25]. The training equipment consisted of a vertical ladder of $1.1 \mathrm{~m}$ height, $0.18 \mathrm{~m}$ width, and $2.0 \mathrm{~cm}$ between the steps at an $80^{\circ}$ slope; at the apex, there was a box with dimensions of $20 \mathrm{~cm} \times 20 \mathrm{~cm} \times 20 \mathrm{~cm}$. The load used was fixed to a metal hook and secured by adhesive tape to the proximal part of the animal's tail. The climbing from the base to the top of the vertical ladder characterized a completed set of exercise. All animals were familiarized with the training protocol for three nonconsecutive days and performed the maximum load-carrying test (MLCT) in the following week. The MLCT consisted of one or more series of climbing with the first one using a load equivalent to $50 \%$ of the body weight. After each set, $30 \mathrm{~g}$ was added until the animal was not able to complete the climbing. The highest weight successfully carried throughout the vertical ladder was considered the maximum load (ML), which was used for the prescription of training intensities and load readjustments. At the end of the protocol, all animals performed the MLCT one last time to evaluate the evolution of strength during the training period.

Strength training was performed three times a week for ten weeks. The protocol consisted of four sets with $50 \%, 75 \%$, $90 \%$, and $100 \%$ intensity of the maximum preestablished load. After performing four sets, the animals were submitted to a $5^{\text {th }}$ set with $100 \%$ of the maximum preestablished load plus $30 \mathrm{~g}$, in order to follow the evolution of the strength gains and adjust the weight. The recovery interval between sets was 60 seconds.

In order to evaluate strength gains during the training protocol, the following parameters were analyzed: absolute (g) and relative loads; for comparison of the results obtained between MLCTs, force gain (g) was calculated using the following formula: final MLCT- initial MLCT.

2.7. Nutrition Assessment. To evaluate the nutritional status, food consumption, caloric intake, protein intake, food efficiency, body weight, body fat, and adiposity index were used. Caloric intake was obtained through the food consumption multiplied by the energetic value of the diet corresponding to the group (standard or high-fat diet). To calculate protein intake, the amount of protein (\%) of the food pellet was calculated from total food intake (g). Food efficiency, which aims to evaluate the animal's ability to convert the energy consumed into body weight, was calculated by dividing the total weight gain of the animals by the total energy intake [26]. The animals' body weight was measured weekly. Body fat was calculated from the sum of the deposits of epididymal, retroperitoneal, and visceral fat. The adiposity index was calculated by dividing the BF by the final body weight (FBW) and multiplying by 100 [27, 28].

2.8. Glucose Tolerance Test. After a 6-hour fasting period, blood was immediately collected from the caudal artery for the evaluation of glucose tolerance. $25 \%$ glucose solution (Sigma-Aldrich, St Louis, MO, USA) was administered intraperitoneally with a dose of $2 \mathrm{~g} / \mathrm{kg}$ of body weight. Blood samples were collected at time points 0 (baseline) and after $30,60,90$, and 120 minutes of glucose infusion. The measurement of glycemic levels was performed with a handheld glucometer (Accu-Chek Go Kit, Roche Diagnostic Brazil Ltda., São Paulo, Brazil). Glucose tolerance in these animals was assessed by the area under the curve (AUC) for glucose.

2.9. Sample Collection and Storage. After the last training session $(48 \mathrm{~h})$, animals were anesthetized with ketamine hydrochloride $\left(50 \mathrm{mg} / \mathrm{kg}\right.$ IP; Dopalen ${ }^{\circledR}$, Sespo Indústria e Comércio Ltda-Vetbrands Division, Jacareí, São Paulo, Brazil) and xylazine hydrochloride $(10 \mathrm{mg} / \mathrm{kg}$ IP, Anasedan ${ }^{\circledR}$, Sespo Indústria e Comércio Ltda-Vetbrands Division, Jacareí, São Paulo, Brazil). For this procedure, the animals were fasted for 12 to 15 hours and euthanized by abdominal incision, with subsequent removal of the heart.

The plantaris and flexor hallucis longus (FHL) muscles from the left hindlimb were removed. Muscles were weighed, and parts of the muscles were placed in falcon tubes containing $10 \%$ formaldehyde solution and phosphate-saline buffer for material fixation and histological analysis. The tibia was also removed and subsequently measured with a 6inch steel analog caliper (Zaas Precision, Amatools Comercial e Importadora Ltda, Piracicaba, São Paulo, Brazil) in order to obtain its length. The weight of the plantaris and FHL muscles were normalized by the tibia length for the purpose of evaluating the skeletal muscle hypertrophy.

2.10. Histological Analysis. For preparation of the histological slide, the formaldehyde-fixed material was subjected 
to a series of steps: (1) dehydration: for removal of water from tissues and replacement by alcohol; (2) diaphanization: to replace the alcohol present in the tissues with xylolene; (3) impregnation: to replace xylene with paraffin; (4) inclusion: for the material to be included in rectangular paraffin blocks; (5) cutting: the paraffin blocks, containing the included tissues, were cut with 5 micrometers thickness in the microtome (RM 2125 RT-Leica, Germany). The slices were transferred to appropriate glass slides and subsequently stained with hematoxylin and eosin (HE). Slides were photographed with an optical microscope and objective lens magnification of 20x and analyzed using the Image-Pro Plus software, version 3.01. The mean fiber crosssectional area (CSA) was determined by measuring the circumference of up to 150 adjacent fibers from the center of each slide.

2.11. Lipid and Hormonal Profile. Serum triglycerides (TG), total cholesterol, and high-density lipoprotein (HDL) levels were determined through specific kits (Bioclin Bioquímica ${ }^{\circledR}$, Belo Horizonte, Minas Gerais, Brazil) and analyzed by automated biochemical equipment BS-200 (Mindray do Brazil, São Paulo, Brazil). Leptin level was determined using an enzyme-linked immunosorbent assay (ELISA) using specific kits (Linco Research Inc., St. Louis, MO, USA). The reading was carried out using a microplate reader (Asys Expert Plus Microplate Reader, Cambourne, Cambridge, UK).

2.12. Statistical Analysis. The results found before training were submitted to Student's $t$ test. For the evaluation of the body weight, two-way ANOVA with repeated measures for the time factor was used, complemented with the Bonferroni post hoc test. For the comparisons between the groups at the end of the experiment, two-way ANOVA was used, supplemented with a Bonferroni post hoc test for normal distribution data and a Holm-Sidak post hoc test for nonparametric samples. Results were expressed as mean\pm standard deviation (SD) and/or median \pm interquartile range. Statistical conclusions were discussed at a significance level of 5\%. For statistical analysis, the programs SigmaStat 3.5 and GraphPad Prism version 6.01 were used.

\section{Results}

3.1. Effect of Induction and Maintenance of Obesity. Body weights were different between group $\mathrm{C}$ and $\mathrm{Ob}$ from the second week of treatment onwards, characterizing the end of the induction period and the beginning of the maintenance period. The body weight difference between groups was maintained throughout the maintenance of obesity, which consisted of 14 weeks (Figure 2).

In relation to dietary intake, no difference was found between groups. However, a higher caloric intake was observed in $\mathrm{Ob}$ animals compared to $\mathrm{C}$ during the initial 16 weeks of the experimental protocol. Although there was no statistical difference in feed efficiency $(p=0.07)$, the treatment was efficient for body weight gain since weight gain in $\mathrm{Ob}$ animals was higher than in $\mathrm{C}$ animals (Table 1).

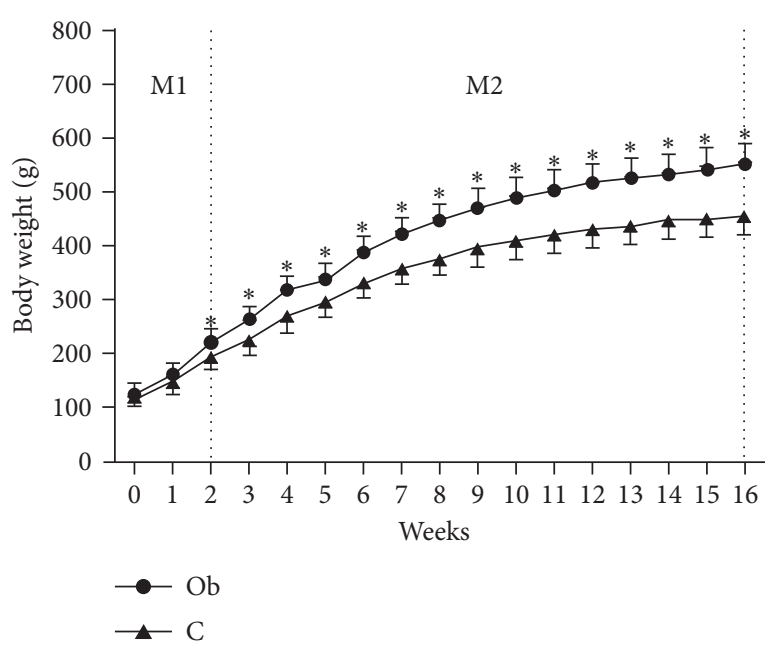

FIGURE 2: Evaluation of body weight during 16 weeks of experimental protocol. Data expressed as mean \pm standard deviation. $\mathrm{C}=$ control $(n=20) ; \mathrm{Ob}=$ obese $(n=16) .{ }^{*} p<0.05$ vs C. Two-way ANOVA for repeated measurements, complemented with the Bonferroni post hoc test.

TABle 1: Nutritional profile during the 16-week experimental protocol prior to strength training.

\begin{tabular}{lcc}
\hline & \multicolumn{2}{c}{ Group } \\
& $\mathrm{C}$ & $\mathrm{Ob}$ \\
\hline Food consumption (g/day) & $22.08 \pm 1.8$ & $21.74 \pm 6.1$ \\
Caloric intake (kcal/day) & $64.47 \pm 5.3$ & $79.35 \pm 6.1^{*}$ \\
Food efficiency (\%) & $4.54 \pm 0.3$ & $4.80 \pm 0.4$ \\
Body weight gain (g) & $331.65 \pm 37.4$ & $425.83 \pm 35.1^{*}$ \\
\hline
\end{tabular}

Data expressed as mean $\pm \mathrm{SD} . \mathrm{C}=\operatorname{control}(n=20)$ and $\mathrm{Ob}=$ obese $(n=16)$. ${ }^{*} p<0.05$ vs. C.

3.2. Effects of Training. Figure 3 shows the evolution of the body weight during the 10 weeks of strength training. Body weight of the ObS and ObT groups was higher compared to the CS and CT groups, respectively, throughout the training period. From the $5^{\text {th }}$ week of strength training onwards, ObT groups presented lower body weight when compared to the ObS. In relation to nutritional parameters during the training protocol ( $17^{\text {th }}$ to $26^{\text {th }}$ weeks), no difference in food consumption was found among groups. However, caloric intake (ObT: $77.5 \pm 4.6$ vs. CT: $62.9 \pm 7.5$; $p<0.05$ ) and feed efficiency (ObT: $0.70 \pm 0.48$ vs. CT: $0.19 \pm 0.34 ; p<0.05$ ) were higher in $\mathrm{ObT}$ when compared to CT. In addition, similar behavior was found in caloric intake (ObS: $82.1 \pm 6.7$ vs. CS: $64.2 \pm 6.2 ; p<0.05$ ) and feed efficiency (ObS: $1.6 \pm 0.3$ vs. CS: $0.69 \pm 0.42, p<0.05)$ of the obese sedentary group. Moreover, besides similar caloric intake between the ObS and the ObT during training, there was significant difference in feed efficiency (ObS: $1.6 \pm 0.3$ vs. ObT: $0.70 \pm 0.48$, $p<0.05$ ), demonstrating the effectiveness of training on reduction of feed (data not shown).

At the beginning of the training protocol, the groups showed no difference in relation to the absolute load. 


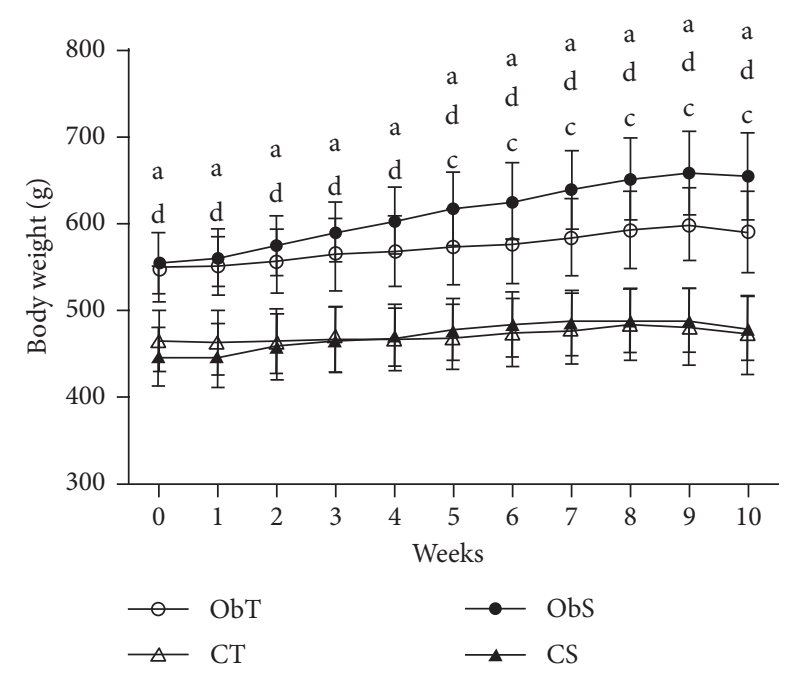

FIgURE 3: Evaluation of body weight during the training period (10 weeks). Data expressed as mean \pm standard deviation. $\mathrm{CS}=$ sedentary control $(n=10)$; $\mathrm{CT}=$ control submitted to the strength training protocol $(n=10) ; \mathrm{ObS}=$ sedentary obese $(n=7) ; \mathrm{ObT}=$ obese submitted to strength training protocol $(n=9) . p<0.05$ : ${ }^{\mathrm{a}} \mathrm{ObS}$ vs CS; ${ }^{\mathrm{b}} \mathrm{CT}$ vs. CS; ${ }^{\mathrm{C}} \mathrm{ObT}$ vs. ObS; ${ }^{\mathrm{d}} \mathrm{ObT}$ vs. CT. Two-way ANOVA for repeated measure, complemented with the post hoc Bonferroni test.

However, the ObT group had a lower relative load compared to the CT group; although the ObS group showed no difference in relation to the CS group, a $p$ value of 0.055 was found (Figure 4(a)). After the training period, the trained animals presented higher absolute load and greater force gain compared to their controls (ObT vs. ObS and CT vs. CS, respectively) (Figures $4(\mathrm{~b})$ and $4(\mathrm{c})$ ). Regarding the relative load, the trained groups presented greater load compared to their controls at the end of the experiment; the relative load was lower in the ObT group compared to the CT group (Figure 4(b)). Therefore, strength training was efficient for gaining muscle force; however, obesity seems to promote impairment in strength.

At the beginning and at the end of the training period, the ObS and ObT groups presented higher body weight when compared to their CS and CT controls, respectively. At the end of 10 weeks of strength training, the FBW of the ObT group was lower than the ObS group. Bodyweight gain was higher in the ObT and ObS animals compared to the CT and CS animals, respectively. In addition, body weight gain was lower in the animals that underwent the training protocol (CT vs. CS; ObT vs. ObS). Body fat and adiposity index presented similar responses since the groups exposed to the HFD (ObT and ObS) presented higher values compared to their respective controls, CT and CS. Body fat and adiposity index were lower in the animals exposed to the HFD and submitted to the training protocol (ObT vs. ObS) (Table 2). In addition, the ObS and ObT groups presented higher leptin values in comparison to their respective controls, and the ObT group presented lower values in relation to the ObS. Although there was no difference in glucose levels under baseline condition, ObS and ObT rats presented higher levels of glucose at 60,90 , and 120 minutes in relation to their respective controls (Figure 5(a)). Nevertheless, the ObT was not able to reverse the glucose damage in ObS (Figure 5(a)). In addition, the same behavior was observed for AUC among the groups (Figure 5(b)). Total cholesterol in the ObS group was lower in comparison to the CS, but the triglyceride values were higher in the ObS group; ObT animals presented lower TG compared to the ObS group (Table 2). There was no difference in HDL levels among the groups.

Weight of FHL muscles of animals from the ObT group was higher compared to that from the CT group (Figure 6(a)) without alterations on the FHL/tibia (Figure 6(b)). However, no difference in plantaris weight and plantaris/tibia was observed among groups (Figures 6(d) and 6(e)). One specific alteration was visualized in tibia length since there were differences between $\mathrm{ObT}$ and $\mathrm{CT}$ groups (data not shown). Moreover, cross-sectional areas FHL and plantaris did not present statistical difference between the groups (Figures 6(c) and 6(f)).

\section{Discussion}

The objective of this study was to evaluate the effects of strength training on skeletal muscle morphology and metabolic adaptations in the obesity condition. The main finding is that the strength training did not induce skeletal muscle hypertrophy, although positive metabolic adaptations were observed in these trained animals.

Experimental models that mimic the eating habits of the population have been used to elucidate the mechanisms of obesity and metabolic disorders [29]. Thus, to induce obesity, a hypercaloric and high-fat diet was used, containing $49.2 \%$ of the energy derived from fat. Our results show that treatment was effective in promoting obesity from the $2^{\text {nd }}$ week, corroborating with previous research $[30,31]$. The difference in body weight of the $\mathrm{C}$ and $\mathrm{Ob}$ groups remained throughout the maintenance period to obesity, with body weight of $\mathrm{Ob}$ animals being $21 \%$ higher than $\mathrm{C}$ animals at the end of this period. The body weight gain was $28.39 \%$ in the $\mathrm{Ob}$ group compared to that in the $\mathrm{C}$ group after 16 weeks of experiment. Similarly, Sitnick et al. [32] found a $31 \%$ increase in body weight of animals that were fed a high-fat diet with $45 \%$ fat in relation to control animals after 14 weeks of experiment. At the end of the experiment, the body fat weight was $145.7 \%$ higher in the ObS animals compared to that in the CS group, and for the trained animals, body weight was found to be $93.33 \%$ higher in the ObT group than in the CT group. The adiposity index of the ObS animals was $78.7 \%$ higher compared to that of the CS and $52.1 \%$ higher for the ObT group compared to that of the CT.

The standard diet used in our study had a caloric content of $2.92 \mathrm{kcal} / \mathrm{g}$, while the high-fat diet presented $3.65 \mathrm{kcal} / \mathrm{g}$, representing a $25 \%$ increase in calories, which justifies the highest body weight gain in this group. Although food efficiency did not show any difference at the end of the maintenance period of obesity, the animals' ability to convert the energy consumed into body weight was higher since the $\mathrm{Ob}$ group presented greater weight gain compared to C. Possibly, the efficiency feeding did not present any 


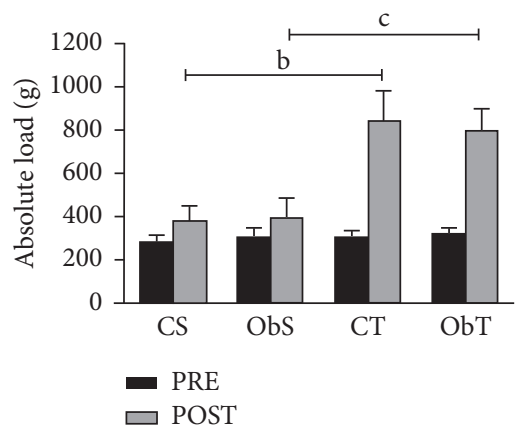

(a)

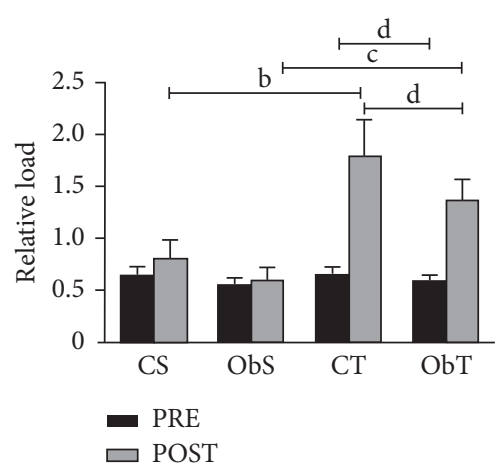

(b)

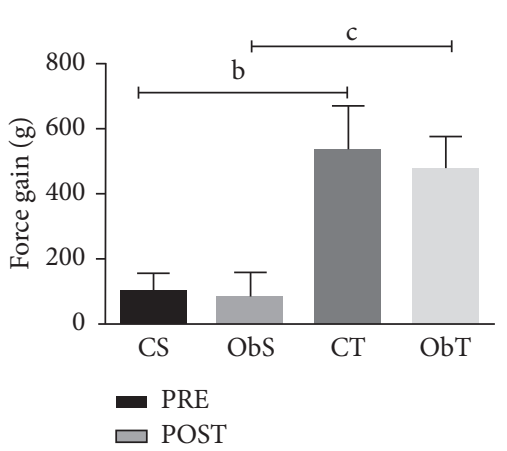

(c)

FIgURe 4: Data expressed as mean \pm standard deviation. $C S=$ sedentary control $(n=10)$; CT $=$ control submitted to the strength training protocol $(n=10)$; ObS = sedentary obese $(n=7)$; ObT = obese submitted to strength training protocol $(n=9)$. $p<0.05$ : ${ }^{\mathrm{b}} \mathrm{CT}$ vs. CS; ${ }^{\mathrm{c}} \mathrm{ObT}$ vs. ObS; ${ }^{\mathrm{d}}$ ObT vs. CT. Two-way ANOVA supplemented with the Bonferroni post hoc test for normal distribution data.

TABLE 2: Adiposity, comorbidities, and hormonal parameters.

\begin{tabular}{|c|c|c|c|c|}
\hline & \multicolumn{4}{|c|}{ Groups } \\
\hline & CS & ObS & $\mathrm{CT}$ & ObT \\
\hline Initial body weight ( $\mathrm{g}$ ) & $447.13 \pm 34.12$ & $555.13 \pm 35.10^{\mathrm{a}}$ & $465.37 \pm 35.97$ & $549.39 \pm 39.50^{\mathrm{d}}$ \\
\hline Final body weight $(\mathrm{g})^{\dagger}$ & $477.97 \pm 36.76$ & $654.31 \pm 50.34^{\mathrm{a}}$ & $471.85 \pm 44.94$ & $590.33 \pm 46.65^{\mathrm{c}, \mathrm{d}}$ \\
\hline Body weight gain (g) & $30.84 \pm 22.80$ & $99.19 \pm 21.37^{\mathrm{a}}$ & $6.48 \pm 18.04^{\mathrm{b}}$ & $40.94 \pm 27.08^{\mathrm{c}, \mathrm{d}}$ \\
\hline Body fat $(\mathrm{g})^{\dagger}$ & $27.75 \pm 5.01$ & $68.2 \pm 13.50^{\mathrm{a}}$ & $27.30 \pm 2.10$ & $52.78 \pm 12.66^{\mathrm{c}, \mathrm{d}}$ \\
\hline Adiposity index (\%) & $5.79 \pm 0.86$ & $10.35 \pm 1.46^{\mathrm{a}}$ & $5.83 \pm 0.73$ & $8.87 \pm 1.71^{\mathrm{c}, \mathrm{d}}$ \\
\hline Leptin $(\mathrm{ng} / \mathrm{mL})^{*}$ & $5.57 \pm 2.98$ & $20.48 \pm 4.35^{\mathrm{a}}$ & $4.77 \pm 2.36$ & $13.24 \pm 7.23^{\mathrm{c}, \mathrm{d}}$ \\
\hline Total cholesterol (mg/dL) & $66.45 \pm 9.10$ & $55.86 \pm 13.18^{\mathrm{a}}$ & $64.05 \pm 9.68$ & $55.00 \pm 8.39$ \\
\hline $\mathrm{HDL}(\mathrm{mg} / \mathrm{dL})$ & $23.30 \pm 3.05$ & $22.86 \pm 6.30$ & $24.70 \pm 3.16$ & $24.06 \pm 3.87$ \\
\hline Triglycerides (mg/dL) & $22.05 \pm 5.99$ & $30.36 \pm 6.90^{\mathrm{a}}$ & $19.60 \pm 3.09$ & $23.06 \pm 9.50^{c}$ \\
\hline
\end{tabular}

Data expressed as mean \pm standard deviation. CS $=$ sedentary control $(n=10) ; \mathrm{CT}=$ control submitted to the strength training protocol $(n=10)$; $\mathrm{ObS}=$ sedentary obese $(n=7) ; \mathrm{ObT}=$ obesity submitted to strength training protocol $(n=9) ;{ }^{\dagger}=$ data presented in median \pm interquartile range. ${ }^{*}$ Number of animals submitted to leptin analysis: CS $(n=9)$; CT $(n=9)$; ObS $(n=7)$; ObT $(n=8) . p<0.05$ : ${ }^{\mathrm{a}} \mathrm{ObS} v s . \mathrm{CT} ;{ }^{\mathrm{b}} \mathrm{CT} v s . \mathrm{CS} ;{ }^{\mathrm{c}} \mathrm{ObT} v s . \mathrm{ObS} ;{ }^{\mathrm{d}} \mathrm{ObT} v s . \mathrm{CT}$.

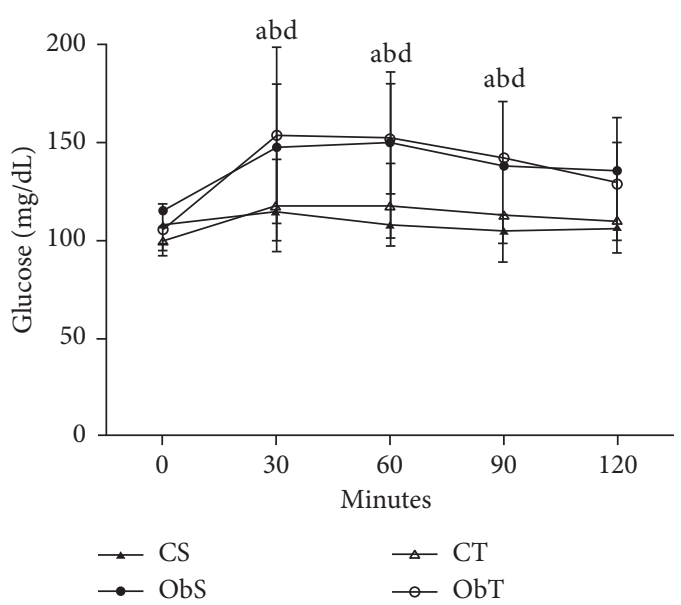

(a)

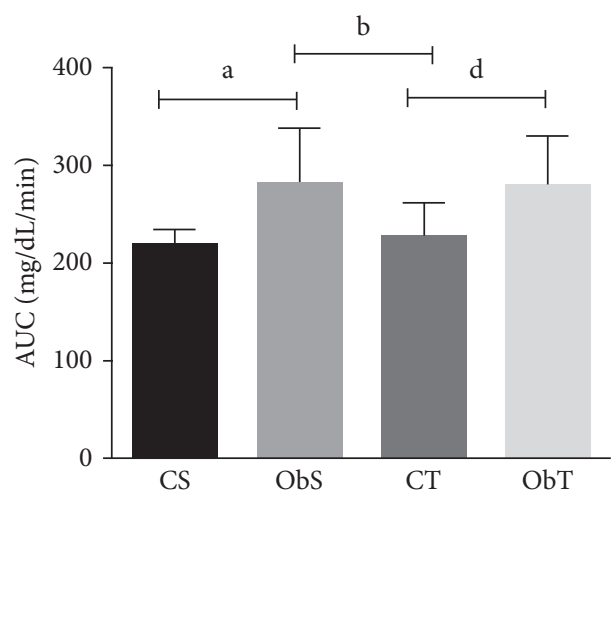

(b)

FIGURE 5: Data expressed as mean \pm standard deviation. CS $=$ sedentary control $(n=10)$; CT $=$ control submitted to the strength training protocol $(n=10)$; ObS = sedentary obese $(n=7)$; ObT = obese submitted to strength training protocol $(n=9) . p<0.05$ : ${ }^{\mathrm{a}} \mathrm{ObS}$ vs. CS; ${ }^{\mathrm{b}} \mathrm{ObS}$ vs. CT; ${ }^{\mathrm{d}}$ ObT vs. CT. Two-way ANOVA supplemented with the Bonferroni post hoc test for normal distribution data. 


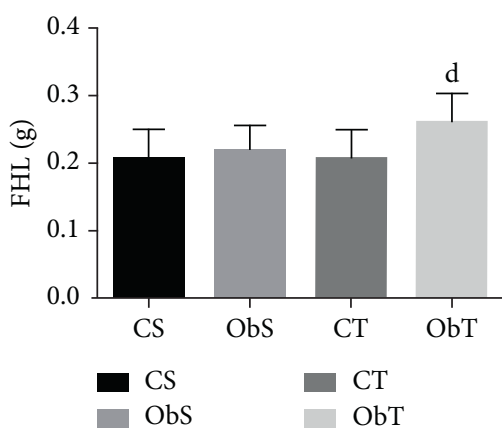

(a)

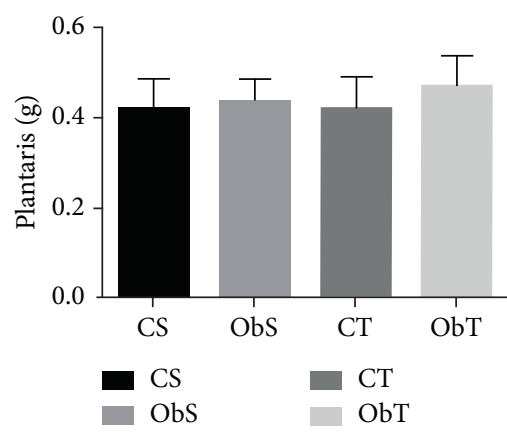

(d)

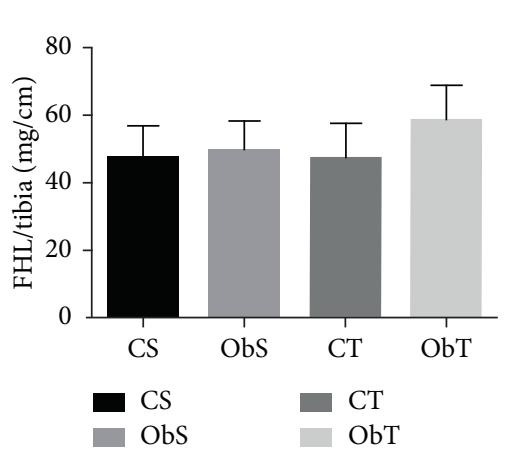

(b)

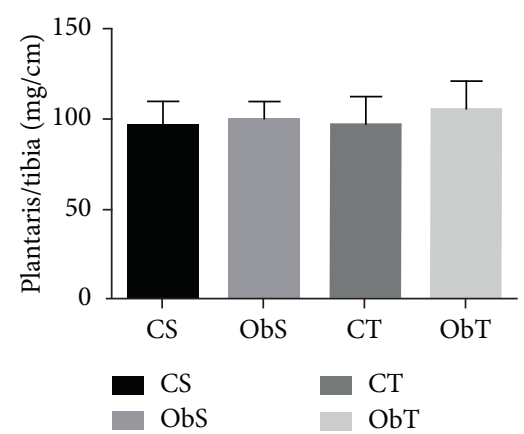

(e)

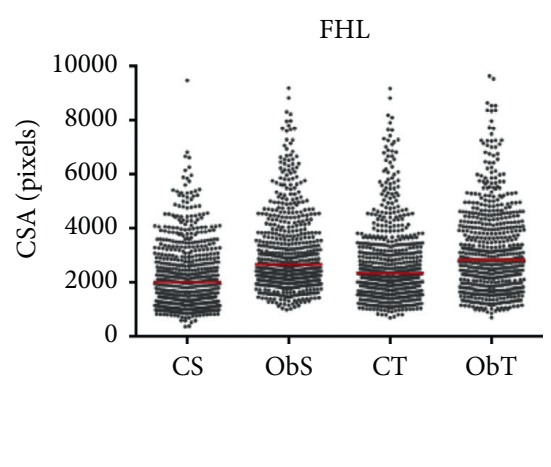

(c)

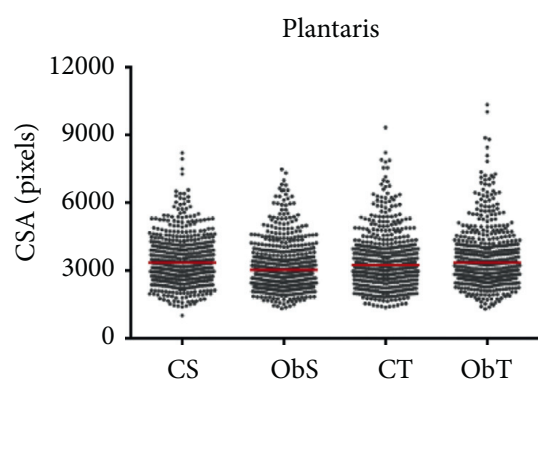

(f)

Figure 6: Morphometric parameters and histological sections of the flexor hallucis longus (FHL) and plantaris muscles. CS = sedentary control $(n=5)$; CT = control submitted to the strength training protocol $(n=5)$; ObS $=$ sedentary obese $(n=5)$; ObT $=$ obese submitted to strength training protocol $(n=5)$. Two-way ANOVA supplemented with the Holm-Sidak post hoc test. $p<0.05:{ }^{\mathrm{d}}$ ObT vs. CT.

difference during the initial 16 weeks of the experimental protocol due to the fact that the rats were still growing during the first weeks [33].

For the biochemical parameters, there were changes under the influence of the HFD since the obesity model promoted glucose intolerance and hypertriglyceridemia in ObS rats, indicating the TG value $37.68 \%$ higher compared to that of the CS group. Thus, the obesity experimental models presented almost all the metabolic disorders, such as glucose intolerance and dyslipidemia; strength training, however, was only able to promote positive changes in TG, which was observed by a reduction in TG levels when compared to the ObS (24.04\%).

The training protocol was efficient to promote positive adaptations in body weight and accumulation of fat in trained animals. As expected, body weight gain was higher in the ObT and ObS groups compared to the CT and CS groups, which may be justified by the higher caloric intake in the animals that were fed a high-fat diet (ObT and ObS). Although there was no difference in caloric intake, body weight gain was lower in the groups submitted to strength training than in the sedentary groups (ObT vs. ObS and CT vs. CS). Body fat and adiposity index also showed that the training was efficient to prevent the effects of the high-fat diet since the ObT group presented lower values for these parameters than the ObS group.
Another important finding is related to the effects of strength training on hormonal regulation. Leptin is an important hormone secreted by adipose tissue that has a role in the control of satiety, commonly found in high concentrations in obese individuals [34]. In the present study, the $\mathrm{ObS}$ and ObT groups presented higher values compared to their respective controls, CS and CT, at 267.68 and $177.56 \%$. In relation to the obesity-induced groups, leptin levels were lower in the animals subjected to the strength training protocol. The ObS group presented a leptin concentration $54.68 \%$ greater than the ObT group, which can be justified by the body fat weight of $12.82 \%$ that was higher in the ObS animals. High blood leptin concentration and resistance to its action are noted occurrences in obesity [35]. Our findings suggest that obesity promoted leptin resistance to action, since even with high levels of leptin, food intake between groups was similar.

The training protocol used was the vertical ladder climbing model with the overload principle as it is the model that most resembles the physiological adaptations found in strength training in humans $[12,25,36]$. At the end of the training period, the groups were fed a high-fat diet presented with higher body weight in comparison to the $\mathrm{C}$ groups (ObT vs. CT and ObS vs. CS). From the $5^{\text {th }}$ week of training, the ObT group had lower body weight compared to the ObS group, showing that the training prevented the gain of body 
weight promoted by the high-fat diet. In addition, the weight of the soleus, tibial, and FHL muscles was higher in the ObT group, $19.5 \%, 15.8 \%$, and $26.1 \%$, respectively, compared to those of the CT group, indicating a possible combined effect of obesity and strength training for gain of muscle mass. However, no difference was observed in muscle hypertrophy between the ObT and CT groups in the AST of plantaris and FHL muscles (Figures 5(c) and 5(f)).

Other authors, using the stair climbing training model, also did not find changes in the skeletal muscle mass [36, 37]. Variations between the analyzed muscles, equipment structure, or training protocol may justify the divergence between the results [37]. As in training for humans, any change in the variables involved in the prescription of the stair climbing training protocol may affect the adaptive response of the animal and, consequently, the results of the study [38].

Although some studies have found skeletal muscle hypertrophy at 8 weeks of training, protocols spanning between 6 and 12 weeks may not be sufficient to produce significant adaptations in skeletal muscle [36]. The duration of the training protocol used lasted 10 weeks and may have an influence on the absence of hypertrophy in the analyzed muscles. In addition, the number of series performed, in which it was established from the results of the pilot study (mean 4.2, climbed per training session), may also have influenced the results. The training model used depends on the voluntary action of the animals, so the number of series performed may have underestimated the physical capacity of the animals; consequently, the muscular stimulation may not have been sufficient to generate hypertrophy.

The absence of muscle hypertrophy in response to the exercise protocol observed in all groups in our study makes it impossible to evaluate the effect of obesity on skeletal muscle hypertrophy induced by strength training. According to Garcia-Vicencio et al. [39] and Bollinger [40], excess body weight can act as a stimulus for chronic overload training promoting favorable muscle adaptations. However, Bollinger [40] points out that the increase in skeletal muscle mass seems to occur only in the muscles bearing the load. The mTOR protein is a key regulator for protein synthesis and skeletal muscle cell hypertrophy [21, 41], and increasing evidence shows dysregulation in the mTOR signaling pathway in diet-induced obesity models [32, 42, 43].

Although the training did not promote changes in muscle mass gain, a force gain was observed in the trained groups (CT vs. CS and ObT vs. CS). At the end of the protocol, the absolute strength of the CT group increased by $172.55 \%$ and of the ObT group by $149.06 \%$. The increase in strength is not necessarily related to the increase in the area of muscle fibers, and the result found may be associated with the neural adaptations that occur with the overload stimulus [44]. Furthermore, at the beginning of the training period, the ObT group had lower relative strength compared to the CT group, and although the ObS group showed no difference in relation to the CS group, a $p$ value of 0.055 was found. At the end of this period, the trained groups presented greater relative strength compared to their controls (CT vs. CS and ObT vs. ObS). The relative strength of the ObT group was also lower compared to that of the CT group. In obesity, the increase in fat mass is greater than the increase in skeletal muscle mass, which means that skeletal muscle represents a smaller percentage of the total body mass. The relative decrease in muscle mass may partly explain the decrease in relative strength found in obesity [40]. However, recent studies suggest that the ability to produce skeletal muscle strength decreases in obesity $[45,46]$. According to Gadducci et al. [47], the functional capacity of skeletal muscle can be assessed from its ability to produce force. Thus, obesity decreased the functional capacity of skeletal muscle in response to strength training in the present study.

The increase in skeletal muscle mass promotes an increase in the basal metabolic rate, contributing to the daily energy expenditure and metabolic health $[8,48]$. However, as the muscles analyzed did not present hypertrophy, it is probable that the results found are justified by the higher energetic expenditure promoted by physical exercise and by the EPOC effect promoted by high-intensity training $[49,50]$.

\section{Conclusions}

The strength training protocol promotes strength gains, but it did not cause skeletal muscle hypertrophy in obesity induced by unsaturated high-fat diet. However, strength training induced positive alterations on adiposity and comorbidities such as reduction in body weight, body fat content, adiposity index, and plasma triglycerides, as well as leptin levels independent of skeletal muscle hypertrophy. These results suggest that high-intensity strength training may be a relevant complementary strategy in combination with nutritional and pharmacological interventions for the treatment of obesity.

\section{Data Availability}

The data supporting the current study are available from the corresponding author upon request.

\section{Conflicts of Interest}

The authors declare that they have no conflicts of interest.

\section{Acknowledgments}

We are grateful to Priscilla Spadeto Altoé for her assistance. This work was supported by the Fundação de Amparo à Pesquisa e Inovação do Espírito Santo-FAPES (grant numbers: $74434489 / 16$ and 67655602/15) - and partially financed by the Coordination for Improvement of Higher Education Personnel-Brazil (CAPES)-Finance Code 001.67655602/15.

\section{References}

[1] World Health Organization, Obesity and Overweight, World Health Organization, Geneva, Switzerland, 2018, http://www. who.int/news-room/fact-sheets/detail/obesity-andoverweight. 
[2] K. R. Fontaine, D. T. Redden, C. Wang, A. O. Westfall, and D. B. Allison, "Years of life lost due to obesity," JAMA, vol. 289, no. 2, pp. 187-193, 2003.

[3] N. Ouchi, J. L. Parker, J. J. Lugus, and K. Walsh, "Adipokines in inflammation and metabolic disease," Nature Reviews Immunology, vol. 11, no. 2, pp. 85-97, 2011.

[4] P. Poirier, T. D. Giles, G. A. Bray et al., "Obesity and cardiovascular disease: pathophysiology, evaluation, and effect of weight loss: an update of the 1997 American heart association scientific statement on obesity and heart disease from the obesity committee of the council on nutrition, physical activity, and metabolism," Circulation, vol. 113, no. 6, pp. 898-918, 2006.

[5] P. W. F. Wilson, R. B. D’Agostino, L. Sullivan, H. Parise, and W. B. Kannel, "Overweight and obesity as determinants of cardiovascular risk: the Framingham experience," Archives of Internal Medicine, vol. 162, no. 16, pp. 1867-1872, 2002.

[6] O. Ciabattari, A. Dal Pai, and V. Dal Pai, "Efeito da natação associado a diferentes dietas sobre o músculo tibial anterior do rato: estudo morfológico e histoquímico," Revista Brasileira de Medicina Do Esporte, vol. 11, no. 2, pp. 121-125, 2005.

[7] F. L. Torres-Leal, M. D. Capitani, and J. Tirapegui, "The effect of physical exercise and caloric restriction on the components of metabolic syndrome," Brazilian Journal of Pharmaceutical Sciences, vol. 45, no. 3, pp. 379-399, 2009.

[8] J. M. Jakicic, K. Clark, E. Coleman et al., "American college of sports medicine position stand. Appropriate intervention strategies for weight loss and prevention of weight regain for adults," Medicine and Science in Sports and Exercise, vol. 33, no. 12, pp. 2145-2156, 2001.

[9] K. M. Fock and J. Khoo, "Diet and exercise in management of obesity and overweight," Journal of Gastroenterology and Hepatology, vol. 28, no. Suppl 4, pp. 59-63, 2013.

[10] K. Baar and K. Esser, "Phosphorylation of p70(S6k) correlates with increased skeletal muscle mass following resistance exercise," American Journal of Physiology-Cell Physiology, vol. 276, no. 1, pp. C120-C127, 1999.

[11] F. B. Favier, H. Benoit, and D. Freyssenet, "Cellular and molecular events controlling skeletal muscle mass in response to altered use," Pflügers Archiv-European Journal of Physiology, vol. 456, no. 3, pp. 587-600, 2008.

[12] J. Cholewa, L. Guimarães-Ferreira, T. da Silva Teixeira et al., "Basic models modeling resistance training: an update for basic scientists interested in study skeletal muscle hypertrophy," Journal of Cellular Physiology, vol. 229, no. 9, pp. 1148-1156, 2014.

[13] B. J. Schoenfeld, J. Grgic, D. Ogborn, and J. W. Krieger, "Strength and hypertrophy adaptations between low- vs. High-load resistance training: a systematic review and metaanalysis," Journal of Strength and Conditioning Research, vol. 31, no. 12, pp. 3508-3523, 2017.

[14] J. Grgic, L. C. Mcllvenna, J. J. Fyfe et al., "Does aerobic training promote the same skeletal muscle hypertrophy as resistance training? A systematic review and meta-analysis," Sports Medicine, vol. 49, no. 2, pp. 233-254, 2018.

[15] T. Rantanen, T. Harris, S. G. Leveille et al., "Muscle strength and body mass index as long-term predictors of mortality in initially healthy men," The Journals of Gerontology Series A: Biological Sciences and Medical Sciences, vol. 55, no. 3, pp. M168-M173, 2000.

[16] A. Guadalupe-Grau, J. A. Carnicero, A. Gómez-Cabello et al., "Association of regional muscle strength with mortality and hospitalisation in older people," Age and Ageing, vol. 44, no. 5, pp. 790-795, 2015.
[17] E. Stamatakis, I.-M. Lee, J. Bennie et al., "Does strengthpromoting exercise confer unique health benefits? A pooled analysis of data on 11 population cohorts with all-cause, cancer, and cardiovascular mortality endpoints," American Journal of Epidemiology, vol. 187, no. 5, pp. 1102-1112, 2018.

[18] D. Akhmedov and R. Berdeaux, "The effects of obesity on skeletal muscle regeneration," Frontiers in Physiology, vol. 4, p. 371, 2013.

[19] M. Francaux and L. Deldicque, "Exercise and the control of muscle mass in human," Pflügers Archiv-European Journal of Physiology, vol. 471, no. 3, pp. 397-411, 2019.

[20] G. R. Marcotte, D. W. D. West, and K. Baar, "The molecular basis for load-induced skeletal muscle hypertrophy," Calcified Tissue International, vol. 96, no. 3, pp. 196-210, 2015.

[21] S. C. Bodine, T. N. Stitt, M. Gonzalez et al., "Akt/mTOR pathway is a crucial regulator of skeletal muscle hypertrophy and can prevent muscle atrophy in vivo," Nature Cell Biology, vol. 3, no. 11, pp. 1014-1019, 2001.

[22] F. Tremblay and A. Marette, "Amino acid and insulin signaling via the mTOR/p70 S6 kinase pathway. A negative feedback mechanism leading to insulin resistance in skeletal muscle cells," The Journal of biological chemistry, vol. 276, no. 41, pp. 38052-60, 2001.

[23] M. R. Papasani, K. Thornton, Z. Yinggian et al., "Does obesity reduce load-induced muscle hypertrophy?" The Journal of Physiology, vol. 588, no. 11, pp. 1819-1820, 2010.

[24] A. J. Ferron, B. B. Jacobsen, P. G. Sant'Ana et al., "Cardiac dysfunction induced by obesity is not related to $\beta$-adrenergic system impairment at the receptor-signalling pathway," PLoS One, vol. 10, Article ID e0138605, 2015.

[25] T. A. Hornberger and R. P. Farrar, "Physiological hypertrophy of the FHL muscle following 8 weeks of progressive resistance exercise in the rat," Canadian Journal of Applied Physiology, vol. 29, no. 1, pp. 16-31, 2004.

[26] D. P. Relling, L. B. Esberg, C. X. Fang et al., "High-fat dietinduced juvenile obesity leads to cardiomyocyte dysfunction and upregulation of Foxo3a transcription factor independent of lipotoxicity and apoptosis," Journal of Hypertension, vol. 24, no. 3, pp. 549-561, 2006.

[27] B. J. Rolls and D. J. Shide, "The influence of dietary fat on food intake and body weight," Nutrition reviews, vol. 50, no. 10, pp. 283-90, 1992.

[28] B. A. Taylor and S. J. Phillips, "Detection of obesity QTLs on mouse chromosomes 1 and 7 by selective DNA pooling," Genomics, vol. 34, no. 3, pp. 389-398, 1996.

[29] A. M. Matias, W. M. Estevam, P. M. Coelho et al., "Differential effects of high sugar, high lard or a combination of both on nutritional, hormonal and cardiovascular metabolic profiles of rodents," Nutrients, vol. 10, 2018.

[30] R. Buettner, J. Schölmerich, and L. C. Bollheimer, "High-fat diets: modeling the metabolic disorders of human obesity in rodents*," Obesity, vol. 15, no. 4, pp. 798-808, 2007.

[31] C. M. Boustany-Kari, M. Gong, W. S. Akers, Z. Guo, and L. A. Cassis, "Enhanced vascular contractility and diminished coronary artery flow in rats made hypertensive from dietinduced obesity," International Journal of Obesity, vol. 31, no. 11, pp. 1652-1659, 2007.

[32] M. Sitnick, S. C. Bodine, and J. C. Rutledge, "Chronic high fat feeding attenuates load-induced hypertrophy in mice," The Journal of Physiology, vol. 587, no. 23, pp. 5753-5765, 2009.

[33] A. F. Nascimento, M. M. Sugizaki, A. S. Leopoldo et al., "Misclassification probability as obese or lean in hypercaloric and normocaloric diet," Biological Research, vol. 41, pp. 253-259, 2008. 
[34] R. Yang and L. A. Barouch, "Leptin signaling and obesity," Circulation Research, vol. 101, no. 6, pp. 545-559, 2007.

[35] A. B. Crujeiras, M. C. Carreira, B. Cabia, S. Andrade, M. Amil, and F. F. Casanueva, "Leptin resistance in obesity: an epigenetic landscape,” Life Sciences, vol. 140, pp. 57-63, 2015.

[36] N. D. Duncan, D. A. Williams, and G. S. Lynch, "Adaptations in rat skeletal muscle following long-term resistance exercise training," European Journal of Applied Physiology, vol. 77, no. 4, pp. 372-378, 1998.

[37] K. Bennell, C. Page, K. Khan et al., "Effects of resistance training on bone parameters in young and mature rats," Clinical and Experimental Pharmacology and Physiology, vol. 27, no. 1-2, pp. 88-94, 2000.

[38] W. K. Neto, W. A. de Silva, A. P. Ciena, C. A. Anaruma, and E. F. Gama, "Vertical climbing for rodent resistance training: a discussion about training parameters," International Journal of Sports Science, vol. 6, pp. 36-49, 2016.

[39] S. Garcia-Vicencio, E. Coudeyre, V. Kluka et al., "The bigger, the stronger? Insights from muscle architecture and nervous characteristics in obese adolescent girls," International Journal of Obesity, vol. 40, no. 2, pp. 245-251, 2016.

[40] L. M. Bollinger, "Potential contributions of skeletal muscle contractile dysfunction to altered biomechanics in obesity," Gait \& Posture, vol. 56, pp. 100-107, 2017.

[41] T. Schmelzle and M. N. Hall, "TOR, a central controller of cell growth,” Cell, vol. 103, no. 2, pp. 253-262, 2000.

[42] S. Paturi, A. K. Gutta, S. K. Kakarla et al., "Impaired overloadinduced hypertrophy in obese Zucker rat slow-twitch skeletal muscle," Journal of Applied Physiology, vol. 108, no. 1, pp. 7-13, 2010.

[43] Z. Hu, H. Wang, I. H. Lee et al., "PTEN inhibition improves muscle regeneration in mice fed a high-fat diet," Diabetes, vol. 59, no. 6, pp. 1312-1320, 2010.

[44] P. V. Komi, "Training of muscle strength and power: interaction of neuromotoric, hypertrophic, and mechanical factors," International Journal of Sports Medicine, vol. 7, no. Suppl 1, pp. 10-15, 1986.

[45] S. J. Choi, D. C. Files, T. Zhang et al., "Intramyocellular lipid and impaired myofiber contraction in normal weight and obese older adults," The Journals of Gerontology Series A: Biological Sciences and Medical Sciences, vol. 71, no. 4, pp. 557-564, 2016.

[46] H. Rahemi, N. Nigam, and J. M. Wakeling, "The effect of intramuscular fat on skeletal muscle mechanics: implications for the elderly and obese," Journal of The Royal Society Interface, vol. 12, no. 109, 2015.

[47] A. Gadducci, R. de Cleva, G. Santarém, P. Silva, J. Greve, and M. Santo, "Muscle strength and body composition in severe obesity," Clinics, vol. 72, no. 5, pp. 272-275, 2017.

[48] J. R. Speakman and C. Selman, "Physical activity and resting metabolic rate," Proceedings of the Nutrition Society, vol. 62, no. 3, pp. 621-634, 2003.

[49] E. Børsheim, S. Knardahl, A. T. Høstmark, and R. Bahr, "Adrenergic control of post-exercise metabolism," Acta Physiologica Scandinavica, vol. 162, no. 3, pp. 313-323, 1998.

[50] E. E. Børsheim and R. Bahr, "Effect of exercise intensity, duration and mode on post-exercise oxygen consumption," Sports Medicine, vol. 33, no. 14, pp. 1037-1060, 2003. 